\title{
Pratiques
}

Linguistique, littérature, didactique

$169-170 \mid 2016$

Enseignement/apprentissage de la langue, des textes et des discours. 40\&nbspans de Pratiques

\section{Les écrits professionnels dans la formation des adultes faiblement qualifiés : quelle didactique du français?}

Professional Papers in Low-Skilled Adults' Education: Which Learning Methods in French?

Marie-Cécile Guernier, Marie-Hélène Lachaud, Jean-Pierre Sautot et Luciane Boganika

\section{Q OpenEdition}

Journals

Édition électronique

URL : http://journals.openedition.org/pratiques/3043

DOI : $10.4000 /$ pratiques.3043

ISSN : 2425-2042

Éditeur

Centre de recherche sur les médiations (CREM)

\section{Référence électronique}

Marie-Cécile Guernier, Marie-Hélène Lachaud, Jean-Pierre Sautot et Luciane Boganika, «Les écrits professionnels dans la formation des adultes faiblement qualifiés : quelle didactique du français ? », Pratiques [En ligne], 169-170 | 2016, mis en ligne le 30 juin 2016, consulté le 19 avril 2019. URL : http:// journals.openedition.org/pratiques/3043; DOI : 10.4000/pratiques.3043

Ce document a été généré automatiquement le 19 avril 2019.

(c) Tous droits réservés 


\title{
Les écrits professionnels dans la formation des adultes faiblement qualifiés : quelle didactique du français?
}

\author{
Professional Papers in Low-Skilled Adults' Education: Which Learning Methods \\ in French?
}

Marie-Cécile Guernier, Marie-Hélène Lachaud, Jean-Pierre Sautot et Luciane Boganika

\section{Questions de recherche et problématiques didactiques}

1 Comme l'indique l'appel à communication du colloque organisé par la revue Pratiques en 2015, la didactique du français s'est constituée comme champ de recherche d'abord au sein de l'école (primaire et secondaire), et a récemment investi l'enseignement supérieur. Par ailleurs, dans le vaste domaine de l'enseignement du français, les didactiques du français langue maternelle (FLM) et du français langue étrangère (FLE) ont construit des champs distincts, la récente émergence du français langue seconde (FLS) ne constituant pas encore un lieu de rapprochement marquant. Une des conséquences de ces délimitations et partitions au sein de la didactique du français est que l'étude de l'enseignement du français auprès des adultes faiblement qualifiés parvient difficilement à émerger, même si les récents développements du français langue professionnelle (FLP) et du français langue d'intégration (FLI) commencent à rendre visibles les recherches conduites dans ce secteur de la formation linguistique. Pour autant, la tendance de l'époque est toujours aux distinctions et à la sectorisation plutôt qu'aux regroupements et à la recherche de congruences. Or de notre point de vue, le domaine de la formation linguistique des jeunes et des adultes faiblement qualifiés, en raison de ses spécificités (type de public, orientation professionnalisante de l'enseignement du français, place des 
financeurs, organisation pédagogique, profils des formateurs, etc.) peut être pour la communauté des didacticiens du français un espace de questionnements nouveaux, tant en ce qui concerne la définition des objets d'enseignement, les pratiques de formation et d'évaluation que la méthodologie de recherche. Un de nos objectifs consiste donc à développer la didactique du français dans ce secteur particulier de la formation des adultes et plus spécifiquement des adultes faiblement scolarisés et/ou faiblement qualifiés et/ou en situation d'illettrisme. La recherche dont nous rendons compte ici s'inscrit dans des travaux conduits selon les modalités de la recherche-action dans le domaine de la formation aux écrits de ces adultes et s'intéresse spécifiquement à l'enseignement des discours oraux et écrits dans le cadre d'une formation professionnelle au sein des entreprises.

Notre recherche poursuit plusieurs buts :

1. construire une typologie à partir d'un corpus de discours utilisés dans ce type de formation,

2. mettre en évidence les compétences langagières orales que la maitrise de ces écrits professionnels requiert et mobilise,

3. évaluer les potentialités didactiques de discours écrits dans la perspective de construire une continuité dans l'apprentissage de l'oral et de l'écrit,

4. élaborer et expérimenter des séances de formation prenant appui sur le développement des compétences orales pour développer les compétences scripturales.

3 Notre recherche articule deux approches: une approche acquisitionnelle qui interroge l'articulation entre la compétence orale et la compétence écrite dans l'apprentissage de l'écrit par des adultes ayant une maitrise de la lecture/écriture faible; une approche plus résolument didactique qui découle de la précédente et a pour objet de mieux définir la nature didactique des actions de formation aux écrits professionnels.

Dans la mesure où pour des adultes qui maitrisent peu l'écrit, l'apprentissage de l'écrit doit emprunter des voies spécifiques et qui ne sont pas celles employées en FLE ou en didactique scolaire, il convient de re-questionner les objets linguistiques, textuels et discursifs enseignés et à enseigner (Guernier, 2012b). Ainsi dans une recherche précédente (Guernier 2009; Guernier, Lachaud \& Sautot; Lachaud \& Rehaili, 2012) au cours de laquelle nous avons analysé des situations de formation à l'écrit pour ces publics, nous avons constaté que majoritairement :

- les apprentissages de l'oral et de l'écrit ne sont pas articulés ;

- le travail sur l'oral est conçu à partir d'échanges informels pour installer le groupe, créer du lien, etc. et conduit de fait à ne pas instituer un apprentissage de l'oral (Guernier, 2010 ; 2012a);

- l'apprentissage de l'écrit est conçu comme un apprentissage des formes de l'écrit en lien avec des pratiques sociales et/ou professionnelles: remplir un chèque, lire une facture, comprendre sa feuille de paie, etc. et/ou des apprentissages en morphosyntaxe.

Il y a donc une contradiction didactique, pour des personnes en difficulté avec l'écrit, de fonder son enseignement-apprentissage principalement sur celui-ci. D'autant que nous avons constaté que les activités écrites ne prennent que rarement appui sur les compétences langagières orales déjà construites et déjà maitrisées de ces personnes dans leur langue maternelle et dans d'autres langues, dont le français. Or, le plus souvent, les adultes dits en difficultés avec l'écrit disposent, à l'oral, dans leur langue maternelle et quelquefois en français, de compétences développées et maitrisent souvent des outils modernes de communication (Lachaud, 2008 ; 2009 ; 2014 ; Barrot \& Lachaud, 2011). Dans 
sa thèse, M.-H. Lachaud (2011) a ainsi mis en évidence les habiletés littératiques de ces personnes dans le cadre de leur activité professionnelle, en particulier dans le domaine du nettoyage. Ces différents constats nous ont conduits à orienter notre recherche sur l'exploration des conditions et des modalités de l'enseignement-apprentissage de l'écrit à partir du travail sur l'oral.

6 Nous analysons ici des documents «professionnels » pour questionner leurs potentialités à être des supports d'enseignement-apprentissage en posant comme hypothèse que pour mieux enseigner le texte écrit, il est nécessaire de passer par le médium du texte oral.

\section{Cadre théorique}

7 Cette problématique s'appuie sur quatre notions principales : les ordres oral et scriptural, le continuum oral/écrit, la littératie et le texte oral. Selon J. Peytard (1970), la langue se réalise dans deux ordres :

- l'oral et le scriptural constituent fondamentalement «deux ordres de situation et de descriptions linguistiques » selon une opposition d'« ordre de réalisation » des messages ;

- l'ordre oral est «celui dans lequel est situé tout message réalisé par articulation et susceptible d'audition »;

- l'ordre scriptural est «celui dans lequel est situé tout message réalisé par la graphie et susceptible de lecture ».

8 Toutefois, dans sa description de la compétence scripturale et dans son travail sur la didactique de l'écrit, M. Dabène remarque que certaines caractéristiques de la scripturalité se manifestent dans certaines productions orales, comme par exemple les échanges oraux à distance (Dabène, 1987, p. 21 sqq.). Ces principales caractéristiques sont :

1. une absence du non verbal qui ne peut donc compenser l'incompréhension du verbal ;

2. un échange de type monologal qui dissocie production et réception du discours, et qui oblige à produire le message en imaginant la compréhension du récepteur ;

3. une décontextualisation du référent, qui oblige à sa construction abstraite par le moyen d'actes de langage précis : dénommer, situer, décrire, etc. ;

4. une planification et une structuration du message proches de celles de l'énoncé écrit et qui linéarise le discours en évitant les reprises et les corrections, verbalise les déictiques (en lien avec la décontextualisation du référent), construit une organisation cohérente du texte et la cohésion du texte en recourant en particulier aux anaphores.

Ainsi M. Dabène met en évidence l'existence d'un continuum entre oral et écrit, et non une dissociation entre les deux ordres. Toutefois, dans une perspective didactique, il semble pertinent d'interroger cette notion de continuum pour envisager plutôt une inclusion de l'ordre écrit dans l'ordre oral. La notion de continuum suppose en effet une linéarité entre l'oral et l'écrit, or notre hypothèse est plutôt que l'écrit est déjà en partie dans l'oral.

La notion de littératie envisage différemment l'articulation entre l'écrit et l'oral. Prenant appui sur les travaux de J.Goody $(1979 ; 1994)$, J.-P. Jaffré (2004, p. 31) propose cette définition de la littératie :

la littératie désigne l'ensemble des activités humaines qui impliquent l'usage de l'écriture, en réception et en production. Elle met un ensemble de compétences de base, linguistiques et graphiques, au service de pratiques, qu'elles soient 
techniques, cognitives, sociales ou culturelles. Son contexte fonctionnel peut varier

d'un pays à l'autre, d'une culture à l'autre, et aussi dans le temps.

11 Selon cette définition, de nombreuses activités humaines qui nécessitent l'usage de l'écrit mobilisent des compétences qui dépassent largement la maitrise du code écrit mais requièrent des habiletés plus larges et peut-être plus complexes. Or il apparait que parmi ces habiletés dites littératiques, certaines sont communes aux compétences orale et écrite: les compétences pragmatiques, les compétences discursives, certaines compétences relatives à la maitrise du temps et de l'espace.

Concernant le texte oral, J. M. Schaeffer (Ducrot \& Schaeffer, 1972, p. 595) définit le texte :

comme une chaine linguistique parlée ou écrite formant une unité communicationnelle, peu importe qu'il s'agisse d'une séquence de phrases, d'une phrase unique, ou d'un fragment de phrase. La notion de texte ne se situe donc pas sur le même plan que celle de phrase [...]. Les structures textuelles, bien que réalisées par des entités linguistiques, constituent des entités communicationnelles : «Un texte n'est pas une structure systématique immanente, mais une unité fonctionnelle d'ordre communicationnel » (H.F. Plett, 1975). Quant à la relation entre texte et discours, elle dépend évidemment de la définition qu'on donne à ce dernier terme. Si on le définit comme tout ensemble d'énoncés d'un énonciateur caractérisé par une unité globale de thème (topic), on dira qu'il peut soit coïncider avec un texte [...] ; soit se composer de plusieurs textes (dans une conversation, il y a une interaction de deux ou plusieurs discours centrés sur leur thème global respectif et, en général, composés chacun de plusieurs textes, puisque chaque réplique de l'échange constitue une unité communicationnelle, et donc un texte, spécifique).

Cette définition rend la notion valide et nous permet de considérer que le texte oral :

1. est une chaine linguistique

2. possède une unité communicationnelle et une unité thématique

3. peut être un composant d'un discours.

De plus, cette définition, dans la mesure où elle concerne le texte parlé ou écrit, établit des analogies entre les formats oraux des textes et les formats écrits. Elle fonde ainsi l'idée que la maitrise des formats textuels oraux est une composante de la maitrise des formats textuels écrits. L'enseignement-apprentissage du texte, en tant que format linguistique, permettrait d'appréhender les notions de cohésion, cohérence, acceptabilité, valables aussi bien à l'écrit qu'à l'oral.

\section{Analyse du corpus d'écrits}

15 La méthodologie mise en œuvre dans le cadre de cette recherche est double. Elle est premièrement linguistique et descriptive. Pour établir la typologie des écrits professionnels utilisés en formation d'adultes, nous avons recueilli un corpus de documents, d'une part dans les entreprises où exercent les adultes en formation, d'autre part dans les formations mises en œuvre. Ces documents professionnels constituent donc une part de l'environnement de travail des apprenants et, pour partie, des supports de leurs apprentissages. Nous avons analysé ces écrits et élaboré la typologie selon deux perspectives :

- au regard des compétences linguistiques langagières et discursives qu'ils mobilisent dans le but d'identifier la congruence entre les compétences orales et scripturales nécessaires à la maitrise de ces écrits ; 
- au regard de leur potentialité didactique.

partir de cette typologie, nous élaborerons des propositions didactiques qui seron testées dans les formations, selon la méthode de l'observation participante, au cours de laquelle les chercheurs sont partie prenante de la conception didactique et de l'animation pédagogique. travail typologique; et nous présentons l'analyse de deux types d'écrits professionnels utilisés en formation : la liste et la procédure. compose de documents de genres divers ${ }^{1}$. Pour envisager une exploitation didactique de cet ensemble, nous avons réalisé une grille d'analyse en quatre points principaux : la fonction de l'écrit; la production et réception de l'information; les modalités sémiotiques ; les formes linguistiques.

La rubrique «Fonction de l'écrit » renseigne la visée discursive principale du document. Dans la lignée des travaux de J. Austin cette entrée renvoie aussi à des notions de langage opératif VS naturel issues de l'ergonomie (Karsenty \& Falzon, 1993) ou à celle de textualisation qui articule parole et travail (Filliettaz, 2002). Les documents sont classés dans cinq catégories :

\begin{tabular}{|l|l|}
\hline $\begin{array}{l}|l| \\
\text { - Attester } \\
\text { - Adhérer }\end{array}$ \\
\hline Demander \\
\hline - Décrire & $\begin{array}{l}\text { - Rendre compte } \\
\text { - Décrire l'entreprise, le salarié, un objet }\end{array}$ \\
\hline - Prescrire & - Prescrire une attitude, une procédure \\
\hline
\end{tabular}

La rubrique «Production et réception de l'information" s'intéresse à la chaine communicative en lien avec l'emploi des documents en situation de travail. Elle comporte deux niveaux : la production en lien avec le destinateur; la réception en lien avec le destinataire.

Quatre types d'interlocuteurs ont été identifiés : un collègue, un membre de la hiérarchie, un fournisseur ou un usager - voire un client. La plupart des documents sont édités à destination du salarié par la hiérarchie ou par les formateurs. Les documents destinés à une communication partant $d u$ salarié sont essentiellement des formulaires administratifs qui exigent rarement un développement rédactionnel.

La rubrique «Modalités sémiotiques » fait intervenir divers marqueurs: les codes iconographiques et le code alphabétique. La complexité des documents oblige à rendre compte de l'intrication de ces deux dimensions. La dimension iconographique est présente sous la forme :

- de logogrammes ;

- de photographies représentant des objets, des postes de travail, des actions de travail...;

- de schémas ou de plans ;

- d'idéogrammes (par ex. des flèches) ;

- de graphiques. 
La dimension alphabétique se présente sous quatre formes: rédactionnel, formulaire, structure liste ou tableau à double entrée. L'intrication des deux dimensions iconographique et scripturale se fait selon deux principes : la juxtaposition ou l'inclusion. Ce niveau de description ne rend pas pleinement compte de la structure du discours. Les documents prescriptifs juxtaposent fréquemment rédactionnel et image, quand la bande dessinée intègre le rédactionnel à l'image. Dans les deux cas, juxtaposition ou inclusion, il existe une structure englobante. Ainsi les documents rendant compte d'une procédure et utilisant des images proposent fréquemment une succession de paragraphes où sont juxtaposés un fragment rédigé et une image.

Enfin, la rubrique « Formes linguistiques » rend compte de la présence :

- d'un lexique spécialisé lié à la situation de travail ;

- de formes syntaxiques : structures phrastiques complètes, infinitives ou nominales ;

- de la présence d'assertions ou de modalités (pouvoir, vouloir, devoir) qui renvoient à une dimension illocutoire, voire perlocutoire.

\section{Étude de deux documents professionnels contrastés}

La typologie qui précède a vocation à permettre de déterminer comment l'écrit s'insère dans une situation didactique professionnelle qui aurait besoin du langage ou dans une situation de didactique du langage qui se développe dans un environnement professionnel.

\section{Lire comme un professionnel?}

26 L'objectif de l'étude de la fiche «outillage du peintre » est d'interroger son apparente facilité de lecture. Pour cela, le recueil de données est complété par : les grilles d'objectifs rédigées en amont de la formation. Ces grilles sont élaborées par l'organisme pour individualiser les apprentissages et rendre compte des nouveaux acquis ; par le script des séances ; par les supports utilisés dans la formation.

Ce document présente une liste d'éléments linguistiques et iconographiques qui peuvent être répartis en deux catégories : les éléments du péritexte et ceux qui font référence au texte. Le péritexte (titre, logogramme et nom de de l'entreprise) devrait informer le lecteur sur la fonction du document et sur son émetteur. Le texte est composé d'une série qui présente des objets relatifs à l'activité du peintre. Il s'agit d'une juxtaposition d'unités lexicales et de photographies. Les unités lexicales sont des étiquettes nominales sans déterminant. Les objets représentés ne correspondent pas exclusivement à la catégorie annoncée par le titre du document. Il s'agit par exemple de : «lessive», «éponge», « chiffons ». 
Figure 1. Fiche « Outillage du peintre»

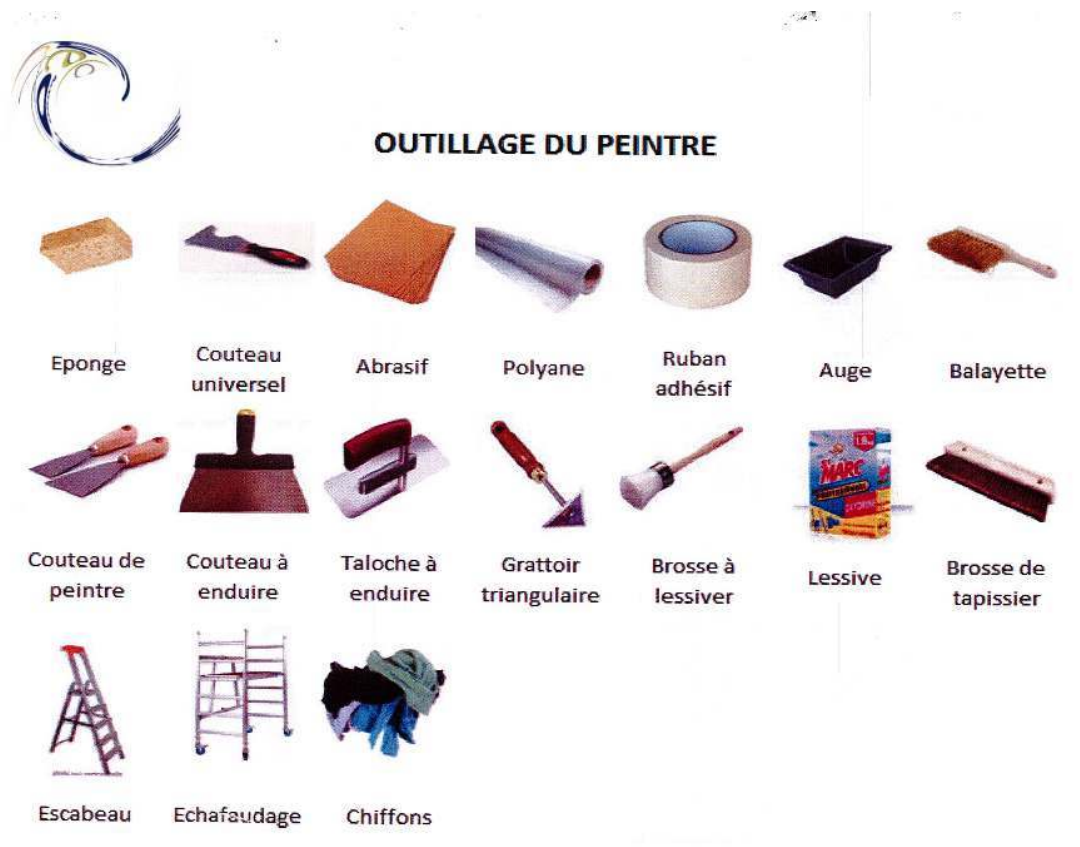
ce que représente l'image, celles correspondant à " auge " ou à "abrasif » en sont des exemples. Parfois, l'image ne permet pas d'identifier le terme inscrit au-dessous ni de relier les objets représentés à leur fonction ni parfois même, de relier les désignations aux objets. La compréhension du document et des termes mentionnés sur celui-ci nécessite à la fois de faire des hypothèses et de chercher d'autres informations pour les mettre en relation entre elles. Enfin, le titre ne renseigne guère le lecteur sur la fonction de ce document dans l'entreprise. La lecture du document peut donc être complexe pour un apprenant aux capacités fragiles. 


\section{Compétences professionnelles reliées à la fiche « outillage du peintre »} mentionnées dans le référentiel métier, alors qu'elles le sont dans le référentiel du CAP dans lequel le vocabulaire technique concerne essentiellement des actions langagières au travail. Précisons que les objectifs de formation ont été déterminés à partir d'un autre cadre de référence, celui des compétences clés en situation professionnelle (ANLCI, 2013). Ce document a notamment été élaboré pour construire des parcours de formation pour tous types de métiers et pour des publics peu qualifiés ni diplômés. Il prévoit un travail spécifique sur le vocabulaire technique. Au vu des référentiels de formation il est donc pertinent d'aborder ce contenu en formation d'adultes. Toutefois, comme le souligne M.C. Guernier (2012a, p. 85), «ce référentiel n'établit donc pas d'articulation entre la construction de connaissances linguistiques et discursives et la construction des compétences langagières et des habiletés littératiques ». Dans la mesure où le référentiel est utilisé pour élaborer les contenus de formation, ceci interroge la manière dont le document «outillage du peintre " peut être utilisé dans sa fonction de support aux apprentissages.

\section{Didactisation de la fiche « outillage du peintre »}

Le document est exploité à deux reprises par le formateur. L'objectif est l'appropriation du lexique professionnel. Les activités de formation ont pour but de permettre aux apprenants débutants à l'oral de comprendre la consigne qui est de rédiger une liste du matériel utilisé sur les chantiers. Le formateur donne la consigne suivante : « Réfléchissez à tout ce que vous utilisez, et après il faut essayer de l'écrire ». Les apprenants travaillent en binôme : un lecteur-scripteur débutant avec un lecteur-scripteur avancé. Plus tard, le formateur propose une activité de classement. Après avoir découpé les images du document, il les mélange à d'autres provenant d'un travail mené en amont, sur le matériel utilisé sur les chantiers. Les stagiaires doivent trouver des critères de classement, puis expliquer et justifier leurs choix de classement. La fiche « outillage du

Pratiques, $169-170 \mid 2016$ 
peintre» est également exploitée dans d'autres activités orales: le «brainstorming lexique ", "se poser des questions sur le lexique professionnel » ou "décrire ses tâches professionnelles ». Le document est donc adapté par le formateur qui élabore un support de formation en vue de développer le lexique professionnel. Le formateur prend également appui sur les ressources que les apprenants mobilisent à l'oral : travail en binôme avec un débutant et un non débutant et en groupe.

Toutefois, lorsqu'on interroge la mise en relation du document avec les situations de travail, on peut se demander si les termes utilisés par les locuteurs dans le travail réel sont ceux que l'on retrouve dans ce document. Une précédente recherche (Lachaud, 2014) a permis de constater que les salariés utilisent leurs propres termes pour décrire le matériel. Ces termes varient d'un lieu à un autre, voire d'une équipe à une autre. C'est le cas de la désignation des balais par les employés du nettoyage qui utilisent «balai pompon» ou «balai espagnol » selon les lieux. Les travaux du réseau «Langage et travail » mettent en évidence les «argots du travail» (Boutet, 2001) qui inscrivent les locuteurs dans une communauté professionnelle voire dans une équipe à travers le langage et les mots partagés. Dans cette continuité, la place du vocabulaire réel et des mots qui sont effectivement utilisés dans les équipes est questionnée : quel vocabulaire faut-il enseigner? Celui qui est prescrit par l'entreprise ou celui qui est effectivement mis en œuvre dans les échanges au travail? Ce qui conduit à interroger la place de l'apprenant dans la formation à visée professionnelle, de ses acquis, de ses pratiques et de sa connaissance de son environnement linguistique professionnel: ses pairs, sa hiérarchie, les locuteurs externes à l'entreprise.

Les documents professionnels constituent un complément sur lesquels les formateurs peuvent prendre appui pour relier les contenus au poste de travail et ainsi favoriser le transfert des nouveaux savoirs. En effet, dans une note de synthèse qui rassemble les travaux des psychologues sur les éléments favorables au transfert de connaissances de la formation vers les situations professionnelles, C. Devos et X. Dumay (2006) montrent que lorsque les contenus sont proches du contexte dans lequel ils sont susceptibles d'être réutilisés, les nouveaux savoirs sont aisés à mettre en œuvre. Cependant, l'usage des documents professionnels pour la formation des adultes ne fait pas l'économie du point de vue du travail réel (Schwartz, 2003) afin d'inclure les situations telles qu'elles seront réellement vécues par les adultes en insertion professionnelle. Ainsi, partir des connaissances et donc de l'expérience des apprenants constituerait un levier. Ceci, afin de permettre l'émergence des acquis mais également de faire des situations professionnelles un objet que l'on peut mettre à distance pour l'interroger et développer de nouvelles capacités d'abstraction.

41 Enfin, nous avons montré que les employés même les moins qualifiés, sont amenés à lire des documents professionnels complexes pour organiser leur travail (Lachaud 2014; 2011). Un travail spécifique sur la lecture permettrait de développer les compétences nécessaires à la lecture des documents professionnels même s'ils paraissent simples comme la fiche "outillage du peintre». Ceci afin de développer des compétences de lecteur-scripteur focalisées sur le document et le texte mais également sur la culture de l'écrit dans le monde professionnel et au-delà. 


\section{Apprendre à dire, ce n'est pas que professionnel}

42 Pour développer des compétences scripturales, il convient, selon nos hypothèses, de repérer au travers des écrits les congruences entre ordres oral et scriptural. Notre but est de déterminer des situations didactiques qui sont propices à l'apprentissage. Pour faire cela, nous nous appuyons sur un second exemple et présentons la page 1 d'un document prescriptif décrivant une procédure d'emballage de pièces mécaniques. Selon notre grille d'analyse, le document est de type prescriptif. Il émane de l'encadrement et est à destination du salarié sur le poste de travail. Il ne requiert aucune production langagière, ni écrite ni orale. On peut estimer que la seule réponse possible est actionnelle : les pièces sont bien emballées. La page est organisée en trois parties :

43 - Le cartouche supérieur identifie l'entreprise au moyen d'un logo. Un titre identifie la nature prescriptive du document et un sous-titre identifie le poste de travail concerné, divers codes identifient le document.

44 - Le pied de page précise la pagination.

45 - Le corps du document présente des photographies prises sur le poste de travail, auxquelles sont juxtaposées des encadrés verbaux numérotés de 1 à 4 . Ces quatre vignettes verbal/image décrivent la procédure à effectuer. L'image y illustre le propos du texte. Une cinquième vignette disposée au centre est descriptive. Le rapport verbal/ image s'y inverse. Sur le plan descriptif, l'image est ici plus explicite que le texte. Au niveau linguistique les cinq vignettes sont hétérogènes. Trois présentent des structures infinitives et deux des structures nominales. Toutes les vignettes expriment de manière implicite une obligation, mais le rapport entre verbal/image est variable. L'action peut être représentée, par exemple :

46 - par les deux modalités sémiotiques (vign.1); par le rédactionnel seul, quand l'image représente l'objet de l'action. (vign.2) ou le produit de l'action (vign.3).

47 Ce matériau varié est exploitable. La question est comment l'exploiter? Grande est la tentation de proposer un questionnaire de lecture, une étude des formes syntaxiques et un relevé lexical qui enrichirait le vocabulaire... Pour l'avoir observé, ce type de propositions pédagogiques centrées sur les codes ne convient guère, en termes d'efficacité, aux volumes des formations ou au niveau de formalisation des apprenants. 

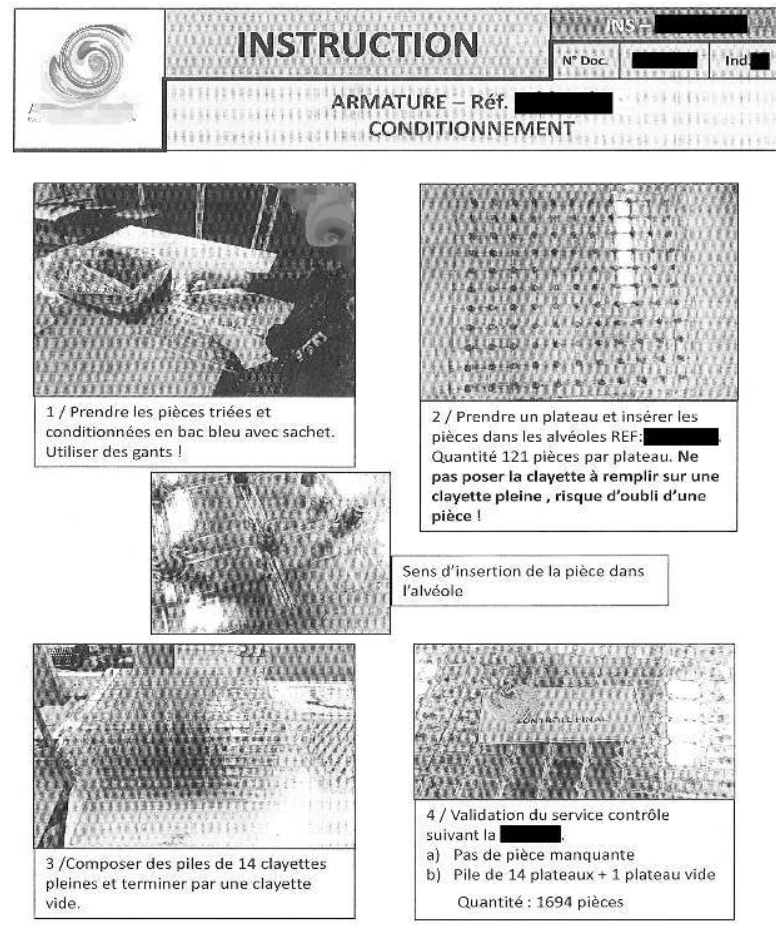

Page $1 / 2$

Plusieurs objectifs pédagogiques peuvent être définis en partant de l'étude d'un tel document. Deux directions de travail sont possibles : de l'oral vers l'écrit et de l'écrit vers l'oral. La familiarité des apprenants avec la situation de travail décrite commandera des configurations pédagogiques variées. Notre propos n'est cependant pas de proposer la configuration d'une situation problème donnée mais de montrer le champ des possibles en termes de congruence discursive entre oral et écrit :

- Une tâche fréquente demandée aux apprenants est de décrire leur poste de travail. Incontestablement ce document participe à cette description. Mais il le fait du seul point de vue de la qualité du produit. Sur le plan langagier, la seule description des gestes prescrits pour parvenir à une production matérielle correcte ne rend pas compte de l'environnement affectif et langagier qui entoure les tâches. Le document est ainsi remarquablement lacunaire sur les motivations des actions qu'il prescrit, sur les difficultés que peut rencontrer l'opérateur, ou encore sur les interactions que génère le contrôle de qualité en aval du poste. Ainsi la verbalisation des non-dits, des ressentis, l'explicitation de la motivation des gestes... constituent une puissante motivation de la production discursive parce qu'il implique l'apprenant.

- La description écrite est strictement monologale quand l'installation sur un poste de travail appelle un dialogue, notamment dans l'intercompréhension des tâches prescrites. La description du poste de travail peut être simulée au cours de dialogues entre un encadrant et un encadré, entre pairs, et cela à plusieurs niveaux : liste des objets présents sur le poste de travail, statuts et fonctions des objets, liste ordonnée des actions à mener, motivation des actions en termes de qualité de la production. La mise en dialogue du discours d'un document par essence monologal contribue à la fois à comprendre mieux les fonctions de cet écrit et sert la lecture. 
- La part descriptive d'un discours sert ce discours quelle qu'en soit la nature. Sa maitrise est donc fondamentale. Le recours à l'image est d'une efficacité certaine sur le plan descriptif, mais il est dommageable sur le plan de la maitrise langagière en incitant à l'usage d'une deixis monstrative. Le travail langagier oral en dehors du poste de travail oblige l'apprenant à verbaliser les conditions de son action sur ce poste, et donc à contextualiser les opérations effectuées. Une des difficultés observées chez certains adultes est une difficulté à expliciter une situation et à en rendre compte de manière compréhensible et/ou exhaustive. L'observation de séances montre que l'interlocuteur résout en partie le problème par une connivence avec l'apprenant. Or si l'on génère une contrainte forte comme l'incompréhension, des difficultés d'explicitation surgissent. D'où l'intérêt de travailler la question de la contextualisation du référent. Cela passe par la mise en œuvre d'opérations descriptives spécifiques : nommer, localiser, qualifier, situer (Charaudeau, 1992, p. 686).

- La difficulté relevée dans la contextualisation est aussi due à une maitrise insuffisante de la planification du discours. La structure de l'écrit proposé pose les bases d'une planification. Cela est particulièrement sensible dans le cas d'une prescription. L'ordre des propos est contraint par la production, oblige à tenir le plan et favorise les opérations sous-jacentes de linéarisation, de mise en mots, d'usages des anaphores et des connecteurs. Le repérage de l'organisation graphique du document est une tâche de lecture qui prépare la production orale, favorise une organisation cohérente du texte oral.

- Le document prescriptif donne matière à une "Grammaire du sens et de l'expression " ${ }^{4}$. Il ne s'agit pas d'étudier la bonne formation morphosyntaxique de telle modalité ou d'étudier la conjugaison des verbes devoir et falloir! L'usage de l'infinitif dans la prescription est une forme tronquée de périphrases verbales. La nominalisation, les formes personnelles de seconde personne (impératif et présent de l'indicatif), l'auxiliarisation sont autant de manières pragmatiques de prescrire ou d'ordonner. Plus largement ce sont les domaines de l'injonction, de l'obligation, de l'interdiction qui sont visées et autant de moyens d'expression qui sont développés, utilisables à l'oral comme à l'écrit.

\section{Conclusion}

Si les documents soumis à nos analyses montrent quelques potentialités didactiques, leur usage en formation continue à questionner leur pertinence. Du point de vue d'une didactique professionnelle, leur forte contextualisation dans une entreprise spécifique questionne le transfert des apprentissages dans d'autres situations. Leurs contenus discursifs peu conséquents ou parfois hors de portée des apprenants interrogent les capacités réellement visées et donc les apprentissages à réaliser : faut-il apprendre à lire et à écrire et si oui quel sens faut-il donner à ces deux termes dans les milieux professionnels concernés?

Du point de vue d'une didactique du langage les perspectives de développement semblent un peu plus prometteuses, notamment en direction du texte oral. Constituer un corpus de discours professionnels permet de relier les écrits à des situations du travail réel et de reconstruire le sens, de l'écrit vers l'oral et inversement. Interroger les écrits du travail prescrit au regard des situations du travail réel donne la possibilité d'aborder les formes discursives (descriptive, injonctive, prescriptive) associées aux documents mais également de développer des habiletés métalinguistiques. Concernant la compétence scripturale, l'apprentissage des codes pourrait se satisfaire de ces documents mais l'apprentissage de la construction du sens d'un discours ne le peut pas. Le risque est alors 
de construire des capacités discursives professionnelles technicistes et désincarnées quand l'enjeu est de permettre l'accès à la culture écrite ou du moins à une culture de l'écrit. Envisager d'autres situations que celles du travail et intégrer les acquis des apprenants dans les contenus constituent alors des pistes à explorer.

\section{BIBLIOGRAPHIE}

ANLCI, (2013). Référentiel des compétences clés en situation professionnelle (RCCSP). En ligne : http:// www.anlci.gouv.fr/Mediatheque/Entreprises/Entreprise/Referentiel-des-competences-cles-ensituation-professionnelle-RCCSP.

BAKHTINE, M. (1984) [1952-1953]. Esthétique de la création verbale. Trad. du russe par A. Aucouturier. Paris : Gallimard.

BARROT, A. \& LACHAUD, M.-H. (2011). « Vers une prise en compte des compétences issues de l'expérience dans les apprentissages en FLP ». Savoirs et Formation, recherches et pratiques 2, p. 41-55.

BOUTET, J. (2001). « La part langagière du travail : bilan et évolution ». Langage \& société 98, p. $17-42$.

CHARAUdeAu, P. (1992). Grammaire du sens et de l'expression. Paris : Hachette Éducation.

DABÈNE, M. (1987). L'adulte et l'écriture: contribution à une didactique de l'écrit en langue maternelle. Bruxelles : De Boeck-Université.

DEVos, C. \& DUMAY, X. (2006). « Les facteurs qui influencent le transfert : une revue de la littérature ». Savoirs 12, p. 11-46.

DUCROT, O. \& SCHAEFFER, J. M. (1972). Nouveau dictionnaire encyclopédique des sciences du langage. Paris : Éd. Le Seuil.

FILLIETTAZ, L. (2002). La parole en action. Québec : Éditions Nota bene.

GOODY, J. (1979). La Raison graphique. La domestication de la pensée sauvage. Paris : Minuit.

- (1994). Entre l'oralité et l'écriture. Paris : Presses universitaires de France.

GUERNIER M.-C. (2009). « Apprendre à lire et à écrire à des adultes ou le rejet du scolaire ». Actes du Symposium International Écoles/cultures École(s) et cultures(s): Quels savoirs? Quelles pratiques? Lille, 12-13 novembre.

- (2010). « Les représentations des formateurs et leurs incidences sur la conception de la formation en linguistique ». Savoirs et formation, recherches et pratiques 1, p. 93-112.

- (2012a). « Les contenus linguistiques dans les référentiels et les discours des formateurs. Définitions, conceptions et références ». Lidil 45, p. 73-92.

- (2012b). «L'apprentissage du français dans les cursus de la formation professionnelle ». In : Dumortier, J. L., Van Beveren, J. \& Vrydaghs, D. (dirs), Quelles progressions curriculaires en français? Namur : Presses universitaires de Namur, p. 67-90.

GUERNIER, M.-C., LACHAUD, M.-H. \& SAUTOT, J. P. (en préparation pour 2016). Former des adultes en difficultés avec l'écrit : questions didactiques. Dijon : Raison et Passion. 
JAFFRÉ, J.-P. (2004). « La littéracie : histoire d'un mot, effets d'un concept ». In : Barré de Miniac, C., Rispail, M. \& Brissaud, C., La littéracie. Conceptions théoriques et pratiques d'enseignement de la lecture-écriture. Paris : L'Harmattan, p. 21-42.

KARSENTY, L. \& FALZON, P. (1993). «L'analyse des dialogues orientés-tâche : Introduction à des modèles de la communication ». In : Six, F. \& Vaxevenoglou, X. (éds), Les aspects collectifs du travail . Toulouse : Octarès.

LACHAUD, M.-H. (2008). « Les salariés en difficulté avec l'écrit : quelles attentes, quelles difficultés, quels besoins de formation ». TransFormations. Recherches en éducation des adultes, 1, p. 37-48. - (2009). «Transfert des nouveaux savoirs et effets de l'écriture sur l'activité des agents de propreté ». Savoirs et formation, recherches et pratiques 1, p. 59-78.

- (2011). Contribution à la formation à l'écrit en milieu professionnel : le cas des métiers de la propreté. Thèse de doctorat, Université de Grenoble.

- (2014). « Du repérage des compétences à la formation professionnelle : le cas des métiers de la propreté ». Le discours et la langue 5, 2, p. 65-76.

LACHAUD, M.-H. \& REHAILI, D. (2012). « Discours des apprenants sur les effets de la formation à l'écrit ». Lidil 45, p. 51-162.

PEYTARD, J. (1970). « Apprentissage du français langue maternelle ». Langue Française 6, p. 35-48.

SCHWARTZ, Y. (2003). « Réflexion autour d'un exemple de travail ouvrier ». In : Schwartz, Y. \& Durrive, L. (éds), Travail et Ergologie. Entretiens sur l'activité humaine. Toulouse : Octarès, p. 38-49.

\section{NOTES}

1. "Genres primaires » selon M. Bakhtine (1984), «modes d'organisation du discours » variés selon P. Charaudeau (1992).

2. Certificat d'aptitude professionnelle.

3. Code ROME répertoire opérationnel des métiers et des emplois.

4. En référence à celle de $\mathrm{P}$. Charaudeau (1992).

\section{RÉSUMÉS}

C'est une contradiction didactique, pour des personnes en difficulté avec l'écrit, de fonder son enseignement-apprentissage principalement sur celui-ci. Nous avons constaté que les activités écrites ne prennent que rarement appui sur les compétences langagières orales déjà construites et déjà maitrisées de ces personnes dans leur langue maternelle et dans d'autres langues, dont le français. Or, le plus souvent, les adultes dits en difficultés avec l'écrit disposent, à l'oral, dans leur langue maternelle et quelquefois en français, de compétences développées et maitrisent souvent des outils modernes de communication. Ces constats nous ont conduits à orienter notre recherche sur l'exploration des conditions et des modalités de l'enseignement- apprentissage de l'écrit à partir du travail sur l'oral. Nous analysons ici des documents "professionnels » pour questionner leurs potentialités à être des supports d'enseignement-apprentissage en posant 
comme hypothèse que pour mieux enseigner le texte écrit, il est nécessaire de passer par le médium du texte oral.

There is a pedagogical conflict for persons experiencing difficulties with writing to base their teaching and learning mostly on the writing. We have found that writing activities are rarely founded on the oral language skills already built and mastered by these persons in their native and other languages, including French. Whereas, the so called low-writing-skilled adults generally have already developed some oral skills in their native language and sometimes in French and they often master modern communication tools. These observations have led us to orient our research and explore terms and conditions of teaching and learning writing from the work on the oral. We are analysing "professional" papers to question their potential to be used as teaching and learning materials and we have a hypothesis that it is necessary to go through the oral text in order to teach better the written text.

INDEX

Mots-clés : littéracie, écrits professionnels, texte oral

Keywords : literacy, professional papers, oral text

\section{AUTEURS}

MARIE-CÉCILE GUERNIER

MARIE-HÉLÈNE LACHAUD

JEAN-PIERRE SAUTOT

LUCIANE BOGANIKA 